\title{
Effect of Trichoderma harzianum and Some Selected Soil Amendment Against Damping off Disease Complex of Potato and Chilli
}

\author{
M. M. Uddin, N. Akhtar, M. T. Islam and A. N. Faruq* \\ Department of Plant Pathology, Sher-e-Bangla Agricultural University, Dhaka-1207, Bangladesh \\ *Corresponding author and Email: nomanfarook@yahoo.com
}

Received: 7 June 2011

Accepted: 26 November 2011

\begin{abstract}
Soil applications with poultry refuse, cocodust, vermicompost, ash, sawdust, khudepana, cowdung, solarized sand, Trichoderma harzianum and or with seed treatment by T. harzianum were evaluated against damping off disease complex of potato and chilli. All the treatments significantly reduced percent damping off over control. T. harzianum treated seed along with soil treatment with $T$. harzianum performed best in terms of seed germination, percent damping off reduction and enhanced growth characters than soil application with $T$. harzianum alone. The experiment indicates that seed treated with $T$. harzianum then sown in different soil amendment applied seed bed performed better in all parameters than only application with soil amendment. Among the different soil amendments, poultry refuse and vermicompost have promising impact on seed germination, reduction of percent damping off and growth of potato and chilli seedlings when applying along with T. harzianum.
\end{abstract}

Keywords: Damping off, potato, chilli, Trichoderma harzianum, soil amendment

\section{Introduction}

Potato (Solanum tuberosum) is one of the most important food crops in Bangladesh, the recorded production was $6648 \mathrm{mt}$. in 401 hectares of land in 2007-2008 (BBS 2009). Depending on quality, 25 to $50 \%$ production cost of potato is required by the cost of seed tubers (Rashid, 1987). By using true potato seed (TPS), seed rate can be reduced from 2.0 tons (tuber seeds) to 100 gm (TPS) per hectare (Chaudhury et al., 1987). Moreover potato tuber carries numerous pathogens including viruses whereas a limited number of diseases are transmitted through true potato seeds (Jones, 1982). These considerations have leaded the International Potato Centre (CIP) along with other research institutes to study the use of true potato seed as an alternative method of potato production. But pre and post emergence damping off disease is the most common problem of potato seedling raised from TPS (True Potato Seed) that affecting seed germination and seedling survival. A number of soil borne pathogens are known to be associated with the disease such as, Fusarium spp.,
Rhizoctonia solani, Pythium spp., Sclerotium rolfsii and Erwinia spp. are predominant (Elango, 1986; Grinstein et al. 1986; Martin and Torres, 1989; Dey, 2005). These organisms have been frequently reported to cause severe reduction in seedling stands of a variety of crop plants (Gammarra et al., 1986). Torres (1989) reported up to $70 \%$ plant loss at CIP research stations due to damping off after transplantation.

Chilii (Capsicum annum) is one of the most important spice crop in the world having nutritive value especially rich in vitamin C. Its green fruits as well as ripe fruits are used as spice for preparing curries, salad etc. and also used in stuffing. Chilli is known to suffer from as many as 83 different diseases, of which more than 40 are caused by fungi (Anon., 1960; Anon., 1966; Rangswami, 1988). Among the fungal diseases, damping off caused by $S$. rolfsii, $F$. oxysporum, Pythium spp., $R$. solani and Phytophthora sp. is the most prevalent in Bangladesh. 
The stated damping off pathogens are world wide in distribution having wide host range and are well known soil-borne pathogens. However, various methods have been suggested to control damping off disease. Cultural practices like management of soil moisture, soil temperature and soil solarization showed promising performance in reducing damping off disease (Das, 1984; Dey, 2005). Application of fungicides against these pathogens may reduce the disease severity but it is neither cost effective nor environment friendly. Available methods reveal that some isolates of Trichoderma harzianum are effective bio-control agent against the above mentioned soil borne pathogens (Roy et al., 1998). The antagonists have also been found to increase the percentage of seedling emergence, plant height, fresh weight and vigour index in different vegetable crops (Begum et al., 1999; Chowdhury et al., 2000; Hossain and Samsuzzaman, 2003; Yeasmin, 2004; Hossain and Naznin, 2005). Organic soil amendment is another important option and eco-friendly approach for controlling damping off disease by developing suppressive nature of soil. Poultry refuse and saw dust proved to be effective for that purpose. (Hilocks and Waller, 1997; Dey, 2005; Islam et al., 2007). Soil amendment such as vermicompost reported to be effective against damping off of chilli seedlings (Szczech, 1999). No reports were found on the simultaneous evaluation of $T$. harzianum and soil amendment as plant growth promoter and suppressive to damping off disease of Potato and chilli. In Bangladesh only soil amendment ability has evaluated to control damping off (caused by Fusarium oxysporum) of eggplant, tomato and chilli (Faruq and Islam, 2007; Islam and Faruq, 2008). The present investigation was designed to study the effect of soil amendment including $T$. harzianum, seed treatment with $T$. harzianum and the combined effect of different soil amendment and seed treatment with $T$. harzianum against damping off disease of potato and chilli.

\section{Materials and Methods}

Field experiment was conducted in seed bed of the farm of Sher-e-Bangla Agricultural University, Dhaka during the period from November, 2008 to January, 2009. Potato variety BARI TPS-1 seeds and Chilli variety BARI Marich 1 (Banglalanka) were collected from BARI (Bangladesh Agricultural Research Institute) Gazipur, and used in the experiment. The treatments of the experiment were; $\mathrm{T}_{1}=$ Soil application of $T$. harzianum $\mathrm{T}_{2}=$ Soil application of poultry refuse, $\mathrm{T}_{3}=$ Soil application of cocodust, $\mathrm{T}_{4}=$ Soil application of vermicompost, $\mathrm{T}_{5}=$ Soil application of ash, $\mathrm{T}_{6}=$ Soil application of sawdust, $\mathrm{T}_{7}=$ Soil application of khudepana (Azolla pinnata), $\mathrm{T}_{8}=$ Soil application of cowdung, $\mathrm{T}_{9}=$ Soil application of solarized sand, $\mathrm{T}_{10}=$ Seed treatment with $T$. harzianum, $\mathrm{T}_{11}=\mathrm{T}_{10}+\mathrm{T}_{1}, \mathrm{~T}_{12}=\mathrm{T}_{10}+\mathrm{T}_{2}, \mathrm{~T}_{13}=\mathrm{T}_{10}$ $+\mathrm{T}_{3}, \mathrm{~T}_{14}=\mathrm{T}_{10}+\mathrm{T}_{4}, \mathrm{~T}_{15}=\mathrm{T}_{10}+\mathrm{T}_{5}, \mathrm{~T}_{16}=\mathrm{T}_{10}+\mathrm{T}_{6}$, $\mathrm{T}_{17}=\mathrm{T}_{10}+\mathrm{T}_{7}, \mathrm{~T}_{18}=\mathrm{T}_{10}+\mathrm{T}_{8}, \mathrm{~T}_{19}=\mathrm{T}_{10}+\mathrm{T}_{9}$ and $\mathrm{T}_{20}=$ Untreated control

The experimental seed bed was prepared by thoroughly ploughing followed by laddering to have a good tilth and the land was properly leveled before sowing. Fertilizers and manures were applied to the seed bed as per recommendation of BARC (1997). Cowdung @ $10 \mathrm{t} / \mathrm{ha}$ was applied during final land preparation. The experiments were laid out in RCBD having three replications for each treatment. The individual seed bed size was $3 \mathrm{~m} \times 1 \mathrm{~m}$. Twenty treatments were evaluated in the experiment as described above and 60 seed bed for potato and 60 seed bed for chilli were used in this study. Block to block distance and seed bed to seed bed distance were $2 \mathrm{~m}$ and $1 \mathrm{~m}$, respectively.

An effective isolate of T. harzianum was used in this experiment. $T$. harzianum was mass multiplied in PDA media incubated at $25^{\circ} \mathrm{C}$ for 7-10 days. Spore suspension were made by scraping the 10-15 days old culture substrate with the help of blender and adjusted the concentration $10^{7}$ conidia/ml solution. Then, soil of the specific plot were drenched with the spore suspension @ 1 lit/pot with the help of compressed air hand sprayer following 
pulverized the soil to mix up the $T$. harzianum spores through out the soil. Seed treatments with bio-agent were done by dipping the seeds in the spore suspension of bio-agent (T. harzianum) for 1 hour. After treatment, the seeds were allowed to dry up for 6 hours. Different soil amendment such as poultry refuse, cowdung, sawdust, ash, khudepana, cocodust, vermicompost, ash and Trichoderma were applied on seed bed soil and keep remain for 15-30 days for proper decomposition, growing antagonistic microorganisms and developing suppressiveness.

Seeds were sown at the rate of $6 \mathrm{~g}$ per seed bed in the field continuously in lines and were covered by soil with the help of hand. Data were recorded on percent germination at 7 days after sowing (DAS), 10 DAS, 13 DAS for Potato and 12 DAS, 15 DAS, 18 DAS for chilli. Damping off disease incidence was recorded at 15, 20, 25 days after sowing for potato and 20, 25, 30 days after sowing for chilli. Seedling growth characters were also recorded such as shoot length, root length, seedling height, fresh shoot weight, fresh root weight, dry shoot weight, dry root weight and biomass at 30 DAS for potato and 35 DAS for chilli. The disease incidence was calculated by the following formula:

$\%$ Disease Incidence $=\frac{\text { Number of infected seedling(s) X } 100}{\text { Number of inspected seedlings }}$

The data on different characters were subjected to statistical analysis using analysis of variance to find out the variation resulting from experimental treatments. The analysis was done using MSTAT computer software. Mean differences among the treatments were compared by Duncan's Multiple Range Test (DMRT).

\section{Results and Discussion \\ 3.1. Treatments performance on germination and damping off of potato}

The effect of seed treatment and soil application of bio-agent (Trichoderma harzianum) and soil amendment on germination percentage of potato at different days after sowing showed significant variation (Table 1). Irrespective days after sowing, the highest germination percentage of potato was recorded in $T_{11}$ (Seed treated with $T$. harzianum + Soil application of $T$. harzianum) where germination percentage was 82.00 at 7 DAS, 87.00 at 10 DAS and 94.33 at 13 DAS followed by $\mathrm{T}_{1}$ (Soil application of $T$. harzianum). On the other hand, the lowest germination percentage at different days after sowing was recorded in $\mathrm{T}_{20}$ (Control) where no treatment was used. At 7 DAS, statistically similar result was found in $\mathrm{T}_{5}, \mathrm{~T}_{16}, \mathrm{~T}_{17}, \mathrm{~T}_{18}$ and $\mathrm{T}_{19}$ in terms of germination percentage. At 10 DAS, $\mathrm{T}_{5}, \mathrm{~T}_{6}, \mathrm{~T}_{7}, \mathrm{~T}_{8}, \mathrm{~T}_{9}, \mathrm{~T}_{10}, \mathrm{~T}_{16}, \mathrm{~T}_{17}$ and $\mathrm{T}_{18}$ showed statistically similar effect in comparison to control $\left(\mathrm{T}_{20}\right)$ in terms of germination percentage. All other soil amendment showed significantly higher germination when seeds were sown in soil amendment applied seed bed after treated with $T$. harzianum than only soil application with different soil amendments.

The effect of seed treatment and soil application of bio-agent (T. harzianum) and soil amendment on percent damping off in potato seedling are also presented in Table 1 . The percent damping off recorded at 15 days after sowing ranged from 0.00 to 8.00 where the highest percent damping off was recorded in $\mathrm{T}_{20}$ (Control) and the lowest in $\mathrm{T}_{11}$ (Seed treated with $T$. harzianum + Soil application of $T$. harzianum). No damping off of potato seedlings was found in soil and seed treated with $T$. harzianum at 15 DAS. Statistically similar result was found in $T_{20}, T_{10}$ and $T_{7}$ in terms of percent damping off recorded at 15 days after sowing. Similar result was also found when percent damping off data were recorded at 20 days after sowing. At 20 days after sowing, statistically similar result was found in $\mathrm{T}_{13}, \mathrm{~T}_{17}$ and $\mathrm{T}_{18}$. The percent damping off of potato seedling varied 2.33 to 28.67 at 25 DAS where the lowest damping off was recorded in soil and seed treatment with T. harzianum and the highest damping off was recorded in control seed bed. Another treatments specially poultry refuse and vermicompost observed better result in reducing percent damping off in comparison to control at different days after sowing. 

Table 1. Impact of different treatments on germination and percent damping off of true potato seedlings at different days after sowing (DAS)

\begin{tabular}{|c|c|c|c|c|c|c|}
\hline \multirow[t]{2}{*}{ Treatments } & \multicolumn{3}{|c|}{ Germination percentage } & \multicolumn{3}{|c|}{ Percent Damping off } \\
\hline & 7 DAS & $10 \mathrm{DAS}$ & 13DAS & 15DAS & 20DAS & 25DAS \\
\hline $\mathrm{T}_{1}$ (Soil application of $T$. harzianum) & $69.00 \mathrm{~b}$ & $75.00 \mathrm{ab}$ & $80.00 \mathrm{~b}$ & $1.67 \mathrm{~h}$ & $3.00 \mathrm{k}$ & 7.001 \\
\hline $\mathrm{T}_{2}$ (Soil application of Poultry Refuse) & $61.67 \mathrm{~cd}$ & $64.67 \mathrm{bc}$ & $71.00 \mathrm{~d}$ & $4.33 \mathrm{~d}-\mathrm{f}$ & $7.00 \mathrm{hi}$ & $13.33 \mathrm{gh}$ \\
\hline $\mathrm{T}_{3}$ (Soil application of Cocodust) & $55.00 \mathrm{e}$ & $60.00 \mathrm{~cd}$ & $66.00 \mathrm{e}$ & $6.00 \mathrm{~d}-\mathrm{f}$ & $12.33 \mathrm{~cd}$ & $17.67 \mathrm{~d}$ \\
\hline $\mathrm{T}_{4}$ (Soil application of Vermicompost) & $60.00 \mathrm{~d}$ & $63.67 \mathrm{bc}$ & $70.00 \mathrm{~d}$ & $4.33 \mathrm{bc}$ & $8.33 \mathrm{gh}$ & $15.00 \mathrm{f}$ \\
\hline $\mathrm{T}_{5}($ Soil application of Ash) & $39.33 \mathrm{gh}$ & $44.00 \mathrm{ef}$ & $51.33 \mathrm{~h}$ & $6.67 \mathrm{~b}-\mathrm{d}$ & $11.00 \mathrm{de}$ & $19.33 \mathrm{c}$ \\
\hline $\mathrm{T}_{6}$ (Soil application of Sawdust) & $34.33 \mathrm{ij}$ & $40.00 \mathrm{ef}$ & $50.00 \mathrm{~h}$ & $5.67 \mathrm{~b}-\mathrm{d}$ & $14.00 \mathrm{~b}$ & $21.33 b$ \\
\hline $\mathrm{T}_{7}$ (Soil application of Khudepana) & $33.00 \mathrm{ij}$ & $36.33 \mathrm{ef}$ & $42.00 \mathrm{i}$ & $7.00 \mathrm{ab}$ & $12.00 \mathrm{~cd}$ & $17.67 \mathrm{~d}$ \\
\hline $\mathrm{T}_{8}$ (Soil application of Cowdung) & $31.67 \mathrm{jk}$ & 34.33 ef & $41.00 \mathrm{i}$ & $6.00 \mathrm{bc}$ & $12.67 \mathrm{bc}$ & $21.00 \mathrm{~b}$ \\
\hline $\mathrm{T}_{9}($ Soil application of Solarized Sand) & $36.00 \mathrm{hi}$ & $41.33 \mathrm{ef}$ & $51.00 \mathrm{~h}$ & $5.00 \mathrm{~cd}$ & $9.33 \mathrm{fg}$ & $17.67 \mathrm{~d}$ \\
\hline $\mathrm{T}_{10}($ Seed treatment of $T$. harzianum $)$ & $33.00 \mathrm{ij}$ & $35.00 \mathrm{ef}$ & $40.00 \mathrm{i}$ & $7.00 \mathrm{ab}$ & $14.00 \mathrm{~b}$ & $22.00 \mathrm{~b}$ \\
\hline $\mathrm{T}_{11}\left(\mathrm{~T}_{10}+\mathrm{T}_{1}\right)$ & $82.00 \mathrm{a}$ & $87.00 \mathrm{a}$ & $94.33 \mathrm{a}$ & $0.00 \mathrm{i}$ & 1.001 & $2.33 \mathrm{~m}$ \\
\hline $\mathrm{T}_{12}\left(\mathrm{~T}_{10}+\mathrm{T}_{2}\right)$ & $64.00 \mathrm{c}$ & $70.00 \mathrm{bc}$ & $77.00 \mathrm{c}$ & $2.67 \mathrm{gh}$ & $5.00 \mathrm{j}$ & $10.33 \mathrm{k}$ \\
\hline $\mathrm{T}_{13}\left(\mathrm{~T}_{10}+\mathrm{T}_{3}\right)$ & $60.00 \mathrm{~d}$ & $65.00 \mathrm{bc}$ & $71.00 \mathrm{~d}$ & $3.67 \mathrm{e}-\mathrm{g}$ & $10.00 \mathrm{ef}$ & $12.67 \mathrm{hi}$ \\
\hline $\mathrm{T}_{14}\left(\mathrm{~T}_{10}+\mathrm{T}_{4}\right)$ & $64.00 \mathrm{c}$ & $48.00 \mathrm{de}$ & $77.33 \mathrm{bc}$ & $3.00 \mathrm{fg}$ & $6.00 \mathrm{ij}$ & $11.33 \mathrm{jk}$ \\
\hline $\mathrm{T}_{15}\left(\mathrm{~T}_{10}+\mathrm{T}_{5}\right)$ & $43.67 \mathrm{f}$ & $49.67 \mathrm{de}$ & $58.00 \mathrm{~g}$ & $5.00 \mathrm{c}-\mathrm{e}$ & $9.00 \mathrm{fg}$ & $15.33 \mathrm{ef}$ \\
\hline $\mathrm{T}_{16}\left(\mathrm{~T}_{10}+\mathrm{T}_{6}\right)$ & $40.00 \mathrm{~g}$ & $45.00 \mathrm{ef}$ & $56.00 \mathrm{~g}$ & 4.00 e-g & $11.00 \mathrm{de}$ & $13.33 \mathrm{gh}$ \\
\hline $\mathrm{T}_{17}\left(\mathrm{~T}_{10}+\mathrm{T}_{7}\right)$ & $38.67 \mathrm{gh}$ & $41.33 \mathrm{ef}$ & $51.33 \mathrm{~h}$ & $5.00 \mathrm{c}-\mathrm{e}$ & $10.00 \mathrm{ef}$ & $14.33 \mathrm{fg}$ \\
\hline $\mathrm{T}_{18}\left(\mathrm{~T}_{10}+\mathrm{T}_{8}\right)$ & $36.33 \mathrm{~g}-\mathrm{i}$ & $39.00 \mathrm{ef}$ & $49.00 \mathrm{~h}$ & $5.00 \mathrm{c}-\mathrm{e}$ & $10.00 \mathrm{ef}$ & $16.33 \mathrm{e}$ \\
\hline $\mathrm{T}_{19}\left(\mathrm{~T}_{10}+\mathrm{T}_{9}\right)$ & $39.67 \mathrm{gh}$ & $48.00 \mathrm{de}$ & $61.00 \mathrm{f}$ & $3.67 \mathrm{e}-\mathrm{g}$ & $7.00 \mathrm{hi}$ & $11.67 \mathrm{ij}$ \\
\hline $\mathrm{T}_{20}$ (Untreated Control) & $28.33 \mathrm{k}$ & $31.00 \mathrm{f}$ & $34.00 \mathrm{j}$ & $8.00 \mathrm{a}$ & $17.67 \mathrm{a}$ & $28.67 \mathrm{a}$ \\
\hline $\operatorname{LSD}(P \leq 0.05)$ & 3.439 & 13.22 & 2.757 & 1.266 & 1.341 & 1.227 \\
\hline
\end{tabular}

Values within the same column having a common letter(s) do not differ significantly $(P \leq 0.05)$ 


\subsection{Impact of different treatments on germination and damping off of chilli}

The effect of seed treatment and soil application of bio-agent ( $T$. harzianum) and soil amendment on germination percentage of chilli at different days after sowing showed significant variation (Table 2). Irrespective days after sowing, the highest germination percentage of chilli was recorded in $\mathrm{T}_{11}$ (Seed treated with $T$. harzianum + Soil application of $T$. harzianum) where germination percentage was 71.33 at $12 \mathrm{DAS}$, 79.33 at 15 DAS and 86.67 at 18 DAS followed by $\mathrm{T}_{12}$ (Seed treated with $T$. harzianum + Soil application of poultry refuse). On the other hand, the lowest germination percentage at different days after sowing was recorded in $\mathrm{T}_{20}$ (Control) where no treatment was used.

The effect of seed treatment and soil application of bio-agent ( $T$. harzianum) and soil amendment on percent damping off in chilli seedling are also presented in Table 2. Irrespective days after sowing, the highest effect against damping off was recorded in $T_{11}$ followed by $T_{1}$ and the lowest effect against damping off was recorded in $\mathrm{T}_{20}$ (Control). Seed treatment and soil application of $T$. harzianum clearly indicates its good effect in reducing pre-emergence and postemergence damping off incidence and increasing seed germination of potato and chilli seedlings. It was also found that the effect against damping off was higher in seed treatment with $T$. harzianum + soil application with $T$. harzianum seed bed than only soil application with $T$. harzianum seed bed. Soil application with soil amendment performances better against damping off when seeds were sown in the seed bed by treated with $T$. harzianum than only soil application with different soil amendment. Similar result was also found in case of germination of potato and chilli seed at different days after sowing.

The findings of the present study are in agreement with the findings of different researchers. Mukhopadhyay (1995) reported that biological seed treatment in tomato, potato, chickpea, lentil and peanut with $T$. harzianum and Gliocladium virens resulted in excellent against a wide range of pathogens including Sclerotium rolfsii and the treatment were consistently as effective as or better than fungicidal seed treatment. Prasad and Gupta (2002) evaluated the bio-efficacy of $T$. harzianum in controlling stem rot of potato caused by $R$. solani. They found that $T$. harzianum significantly inhibited the mycelial growth and sclerotial production of $R$. solani. Manoranjitham et al. (2000) reported that soil application of $T$. viride and Pseudomonas fluorescens effectively controlled the preemergence and post-emergence damping off of tomato caused by Pythium aphanidermatum under pot culture experiments. Islam et al. (2007) reported that poultry refuse suppressed the percent damping off and increased germination of eggplant, tomato and chilli seedlings. Islam and Faruq (2008) reported that poultry refuse reduced the damping off incidence by $100 \%$ at 20 DAS, $94.12 \%$ at 25 DAS and $89.46 \%$ at 30 DAS over control and seedlings growth characters like shoot length, root length, seedling height, fresh shoot weight, fresh root weight and fresh seedling weight of chilli were also enhanced by the application of poultry refuse.

\subsection{Effect of treatments on different growth characters of potato and chilli seedlings}

The effect of $T$. harzianum and some selected soil amendment on different growth characters of potato seedlings were showed significant variation except dry root weight. (Table 3 ). The seedling height of potato seedling ranged from 10.40 to $16.30 \mathrm{~cm}$ where the maximum height was recorded in $\mathrm{T}_{11}$ followed by $\mathrm{T}_{12}$ and the lowest was recorded in $\mathrm{T}_{20}$. In case of shoot length, the highest value was recorded in $T_{11}$ followed by $T_{12}$ and the lowest shoot length was recorded in $T_{20}$. The root length of potato seedling varied 0.85 to $3.65 \mathrm{~cm}$ where the highest root length was recorded in $\mathrm{T}_{11}$ and the lowest in $\mathrm{T}_{20}$. The highest fresh shoot and root weight was recorded in $T_{11}$ which was closely followed by $T_{12}$. No significant difference was found in case of dry root weight but the highest value was recorded in $T_{11}$. 
Table 2. Treatments effect on germination and percent damping off of chilli seedlings at different days after sowing (DAS)

\begin{tabular}{|c|c|c|c|c|c|c|}
\hline \multirow{2}{*}{ Treatments } & \multicolumn{3}{|c|}{ Germination percentage } & \multicolumn{3}{|c|}{ Percent Damping off } \\
\hline & $12 \mathrm{DAS}$ & $15 \mathrm{DAS}$ & 18DAS & $20 \mathrm{DAS}$ & 25DAS & 30 DAS \\
\hline $\mathrm{T}_{1}$ (Soil application of $T$. harzianum) & $64.00 \mathrm{~b}-\mathrm{d}$ & $70.67 \mathrm{~b}$ & $76.67 \mathrm{bc}$ & $1.33 \mathrm{k}$ & 2.331 & $4.67 \mathrm{i}$ \\
\hline $\mathrm{T}_{2}$ (Soil application of Poultry Refuse) & $61.00 \mathrm{f}-\mathrm{h}$ & $64.33 \mathrm{fg}$ & $70.33 \mathrm{fg}$ & $3.67 \mathrm{~g}-\mathrm{i}$ & $5.67 \mathrm{~g}-\mathrm{i}$ & $8.67 \mathrm{gh}$ \\
\hline $\mathrm{T}_{3}$ (Soil application of Cocodust) & $57.67 \mathrm{j}$ & $61.33 \mathrm{i}$ & $67.00 \mathrm{jk}$ & $4.00 \mathrm{f}-\mathrm{h}$ & $6.33 \mathrm{f}-\mathrm{h}$ & $9.33 \mathrm{fg}$ \\
\hline $\mathrm{T}_{4}$ (Soil application of Vermicompost) & $62.33 \mathrm{~d}-\mathrm{fg}$ & $65.67 \mathrm{e}$ & 71.33 ef & $3.67 \mathrm{~g}-\mathrm{i}$ & $5.67 \mathrm{~g}-\mathrm{i}$ & $9.00 \mathrm{gh}$ \\
\hline $\mathrm{T}_{5}$ (Soil application of Ash) & $55.67 \mathrm{k}$ & $59.33 \mathrm{j}$ & $63.67 \mathrm{~m}$ & $5.33 \mathrm{~b}-\mathrm{e}$ & $8.00 \mathrm{c}-\mathrm{e}$ & $11.00 \mathrm{de}$ \\
\hline $\mathrm{T}_{6}$ (Soil application of Sawdust) & $57.33 \mathrm{j}$ & $59.67 \mathrm{j}$ & 65.331 & $5.67 \mathrm{~b}-\mathrm{d}$ & $9.33 \mathrm{bc}$ & $12.33 \mathrm{~cd}$ \\
\hline $\mathrm{T}_{7}$ (Soil application of Khudepana) & $59.67 \mathrm{hi}$ & $63.00 \mathrm{~h}$ & $66.67 \mathrm{jk}$ & $6.33 \mathrm{ab}$ & $8.33 \mathrm{~b}-\mathrm{d}$ & $13.67 \mathrm{bc}$ \\
\hline $\mathrm{T}_{8}$ (Soil application of Cowdung) & $60.67 \mathrm{gh}$ & $62.67 \mathrm{~h}$ & $66.33 \mathrm{kl}$ & 5.67 b-d & $9.67 \mathrm{~b}$ & $15.00 \mathrm{~b}$ \\
\hline $\mathrm{T}_{9}$ (Soil application of Solarized Sand) & $61.33 \mathrm{f}-\mathrm{h}$ & $64.33 \mathrm{fg}$ & $67.67 \mathrm{ij}$ & $5.00 \mathrm{c}-\mathrm{f}$ & $7.33 \mathrm{~d}-\mathrm{f}$ & $11.33 \mathrm{de}$ \\
\hline $\mathrm{T}_{10}($ Seed treatment of $T$. harzianum $)$ & $54.33 \mathrm{k}$ & $60.33 \mathrm{ij}$ & $62.67 \mathrm{~m}$ & $6.00 \mathrm{bc}$ & $9.33 \mathrm{bc}$ & $14.67 \mathrm{~b}$ \\
\hline $\mathrm{T}_{11}\left(\mathrm{~T}_{10}+\mathrm{T}_{1}\right)$ & $71.33 \mathrm{a}$ & 79.33 a & 86.67 a & 0.001 & $0.33 \mathrm{~m}$ & $2.00 \mathrm{j}$ \\
\hline $\mathrm{T}_{12}\left(\mathrm{~T}_{10}+\mathrm{T}_{2}\right)$ & $64.67 \mathrm{bc}$ & $68.67 \mathrm{c}$ & $76.00 \mathrm{c}$ & $2.00 \mathrm{jk}$ & $3.33 \mathrm{kl}$ & $4.67 \mathrm{i}$ \\
\hline $\mathrm{T}_{13}\left(\mathrm{~T}_{10}+\mathrm{T}_{3}\right)$ & $63.67 \mathrm{c}-\mathrm{e}$ & $67.33 \mathrm{~d}$ & $72.67 \mathrm{~d}$ & $3.333 \mathrm{hi}$ & $4.67 \mathrm{i}-\mathrm{k}$ & $7.67 \mathrm{~h}$ \\
\hline $\mathrm{T}_{14}\left(\mathrm{~T}_{10}+\mathrm{T}_{4}\right)$ & $65.33 \mathrm{~b}$ & $70.33 \mathrm{~b}$ & $77.33 \mathrm{~b}$ & $2.67 \mathrm{ij}$ & $4.00 \mathrm{jk}$ & $6.00 \mathrm{i}$ \\
\hline $\mathrm{T}_{15}\left(\mathrm{~T}_{10}+\mathrm{T}_{5}\right)$ & $58.67 \mathrm{ij}$ & $62.67 \mathrm{~h}$ & $68.67 \mathrm{hi}$ & $4.67 \mathrm{~d}-\mathrm{g}$ & 6.67 e-h & $8.67 \mathrm{gh}$ \\
\hline $\mathrm{T}_{16}\left(\mathrm{~T}_{10}+\mathrm{T}_{6}\right)$ & $60.67 \mathrm{gh}$ & $63.33 \mathrm{gh}$ & $69.33 \mathrm{gh}$ & $3.67 \mathrm{~g}-\mathrm{i}$ & $5.33 \mathrm{~h}-\mathrm{j}$ & $8.67 \mathrm{gh}$ \\
\hline $\mathrm{T}_{17}\left(\mathrm{~T}_{10}+\mathrm{T}_{7}\right)$ & $62.00 \mathrm{e}-\mathrm{g}$ & $66.00 \mathrm{e}$ & 70.67 ef & $5.00 \mathrm{c}-\mathrm{f}$ & $7.00 \mathrm{~d}-\mathrm{g}$ & 10.67 ef \\
\hline $\mathrm{T}_{18}\left(\mathrm{~T}_{10}+\mathrm{T}_{8}\right)$ & $62.67 \mathrm{~d}-\mathrm{f}$ & 65.33 ef & $71.67 \mathrm{de}$ & 4.33 e-h & $6.67 \mathrm{e}-\mathrm{h}$ & $11.67 \mathrm{de}$ \\
\hline $\mathrm{T}_{19}\left(\mathrm{~T}_{10}+\mathrm{T}_{9}\right)$ & $63.67 \mathrm{~b}-\mathrm{e}$ & $66.33 \mathrm{de}$ & $71.67 \mathrm{de}$ & $3.67 \mathrm{~g}-\mathrm{i}$ & $5.33 \mathrm{~h}-\mathrm{j}$ & $8.00 \mathrm{gh}$ \\
\hline $\mathrm{T}_{20}$ (Untreated Control) & 52.671 & $55.33 \mathrm{k}$ & $58.67 \mathrm{n}$ & $7.33 \mathrm{a}$ & $13.33 \mathrm{a}$ & $18.67 \mathrm{a}$ \\
\hline $\operatorname{LSD}(P \leq 0.05)$ & 1.506 & 1.127 & 1.174 & 1.076 & 1.274 & 1.410 \\
\hline
\end{tabular}

Values within the same column having a common letter(s) do not differ significantly $(P \leq 0.05)$ 
Table 3. Growth performance of true potato seedlings (TPS) under different treatments practiced

\begin{tabular}{|c|c|c|c|c|c|c|c|c|}
\hline Treatments & $\begin{array}{l}\text { Seedling } \\
\text { height }\end{array}$ & $\begin{array}{l}\text { Shoot } \\
\text { length }\end{array}$ & Root length & $\begin{array}{l}\text { Fresh shoot } \\
\text { weight }\end{array}$ & $\begin{array}{l}\text { Fresh root } \\
\text { weight }\end{array}$ & $\begin{array}{l}\text { Dry shoot } \\
\text { weight }\end{array}$ & $\begin{array}{l}\text { Dry root } \\
\text { weight }\end{array}$ & Biomass \\
\hline $\mathrm{T}_{1}$ (Soil application of $T$. harzianum) & $13.10 \mathrm{bc}$ & $10.13 \mathrm{~b}-\mathrm{d}$ & $2.97 \mathrm{~b}$ & $0.832 \mathrm{bc}$ & $0.053 \mathrm{~b}-\mathrm{d}$ & $0.064 \mathrm{bc}$ & 0.005 & $0.885 \mathrm{bc}$ \\
\hline $\mathrm{T}_{2}$ (Soil application of Poultry Refuse) & 12.75 b-d & $10.29 \mathrm{bc}$ & $2.47 \mathrm{~d}$ & $0.686 \mathrm{c}-\mathrm{f}$ & $0.043 \mathrm{c}-\mathrm{e}$ & $0.056 \mathrm{~b}-\mathrm{d}$ & 0.004 & $0.736 \mathrm{c}-\mathrm{e}$ \\
\hline $\mathrm{T}_{3}$ (Soil application of Cocodust) & $11.22 \mathrm{i}$ & $9.25 \mathrm{f}$ & $1.97 \mathrm{gh}$ & $0.604 \mathrm{~d}-\mathrm{g}$ & 0.026 e-g & 0.049 c-e & 0.003 & $0.638 \mathrm{c}-\mathrm{f}$ \\
\hline $\mathrm{T}_{4}$ (Soil application of Vermicompost) & 12.00 e-h & 9.74 b-f & $2.33 \mathrm{~d}-\mathrm{f}$ & $0.722 \mathrm{c}-\mathrm{f}$ & $0.036 \mathrm{c}-\mathrm{f}$ & $0.053 \mathrm{~cd}$ & 0.004 & $0.771 \mathrm{~b}-\mathrm{e}$ \\
\hline $\mathrm{T}_{5}$ (Soil application of Ash) & $11.48 \mathrm{~h}-\mathrm{i}$ & $9.22 \mathrm{f}$ & $2.27 \mathrm{~d}-\mathrm{g}$ & $0.590 \mathrm{e}-\mathrm{g}$ & $0.028 \mathrm{f}-\mathrm{g}$ & $0.038 \mathrm{~d}-\mathrm{f}$ & 0.002 & $0.627 \mathrm{c}-\mathrm{f}$ \\
\hline $\mathrm{T}_{6}$ (Soil application of Sawdust) & $11.63 \mathrm{f}-\mathrm{i}$ & $9.70 \mathrm{~b}-\mathrm{f}$ & $1.90 \mathrm{~h}$ & $0.624 \mathrm{~d}-\mathrm{f}$ & $0.025 \mathrm{e}-\mathrm{g}$ & 0.050 c-e & 0.001 & $0.674 \mathrm{c}-\mathrm{f}$ \\
\hline $\mathrm{T}_{7}$ (Soil application of Khudepana) & $11.47 \mathrm{~g}-\mathrm{i}$ & $9.62 \mathrm{c}-\mathrm{f}$ & $1.85 \mathrm{~h}$ & $0.548 \mathrm{fg}$ & 0.029 e-g & $0.044 \mathrm{c}-\mathrm{e}$ & 0.003 & $0.577 \mathrm{~d}-\mathrm{g}$ \\
\hline $\mathrm{T}_{8}$ (Soil application of Cowdung) & $11.47 \mathrm{~g}-\mathrm{i}$ & 9.34 ef & $2.13 \mathrm{e}-\mathrm{h}$ & $0.654 \mathrm{c}-\mathrm{f}$ & 0.034 ef & $0.056 \mathrm{~b}-\mathrm{d}$ & 0.002 & $0.687 \mathrm{c}-\mathrm{f}$ \\
\hline $\mathrm{T}_{9}$ (Soil application of Solarized Sand) & $11.47 \mathrm{~g}-\mathrm{i}$ & $9.53 \mathrm{~d}-\mathrm{f}$ & $2.07 \mathrm{f}-\mathrm{h}$ & $0.651 \mathrm{c}-\mathrm{f}$ & $0.032 \mathrm{ef}$ & $0.052 \mathrm{~cd}$ & 0.002 & $0.683 \mathrm{c}-\mathrm{f}$ \\
\hline $\mathrm{T}_{10}($ Seed treatment of $T$. harzianum $)$ & $11.32 \mathrm{hi}$ & $9.87 \mathrm{~b}-\mathrm{f}$ & $1.45 \mathrm{i}$ & $0.427 \mathrm{gh}$ & $0.019 \mathrm{fg}$ & $0.032 \mathrm{ef}$ & 0.002 & $0.447 \mathrm{fg}$ \\
\hline $\mathrm{T}_{11}\left(\mathrm{~T}_{10}+\mathrm{T}_{1}\right)$ & $16.30 \mathrm{a}$ & $12.65 \mathrm{a}$ & $3.65 \mathrm{a}$ & $1.22 \mathrm{a}$ & $0.073 \mathrm{a}$ & $0.084 \mathrm{a}$ & 0.009 & $1.289 \mathrm{a}$ \\
\hline $\mathrm{T}_{12}\left(\mathrm{~T}_{10}+\mathrm{T}_{2}\right)$ & $13.25 \mathrm{~b}$ & $10.35 \mathrm{~b}$ & $2.90 \mathrm{~b}$ & $0.931 \mathrm{~b}$ & $0.066 \mathrm{ab}$ & $0.074 \mathrm{ab}$ & 0.007 & $1.00 \mathrm{~b}$ \\
\hline $\mathrm{T}_{13}\left(\mathrm{~T}_{10}+\mathrm{T}_{3}\right)$ & $11.75 \mathrm{f}-\mathrm{i}$ & $9.50 \mathrm{~d}-\mathrm{f}$ & $2.25 \mathrm{~d}-\mathrm{g}$ & $0.668 \mathrm{c}-\mathrm{f}$ & $0.029 \mathrm{e}-\mathrm{g}$ & $0.056 \mathrm{~b}-\mathrm{d}$ & 0.004 & $0.697 \mathrm{c}-\mathrm{f}$ \\
\hline $\mathrm{T}_{14}\left(\mathrm{~T}_{10}+\mathrm{T}_{4}\right)$ & $12.53 \mathrm{c}-\mathrm{e}$ & $9.77 \mathrm{~b}-\mathrm{f}$ & $2.77 \mathrm{bc}$ & $0.792 \mathrm{~b}-\mathrm{d}$ & $0.055 \mathrm{bc}$ & $0.060 \mathrm{bc}$ & 0.006 & $0.858 \mathrm{~b}-\mathrm{d}$ \\
\hline $\mathrm{T}_{15}\left(\mathrm{~T}_{10}+\mathrm{T}_{5}\right)$ & 11.93 e-h & 9.41 ef & $2.53 \mathrm{~d}-\mathrm{g}$ & $0.696 \mathrm{c}-\mathrm{f}$ & $0.031 \mathrm{ef}$ & $0.046 \mathrm{c-e}$ & 0.003 & $0.728 \mathrm{c}-\mathrm{e}$ \\
\hline $\mathrm{T}_{16}\left(\mathrm{~T}_{10}+\mathrm{T}_{6}\right)$ & $12.27 \mathrm{~d}-\mathrm{f}$ & 10.14 b-d & $2.12 \mathrm{e}-\mathrm{h}$ & $0.698 \mathrm{c}-\mathrm{f}$ & $0.041 \mathrm{c}-\mathrm{e}$ & $0.053 \mathrm{~cd}$ & 0.003 & $0.739 \mathrm{c}-\mathrm{e}$ \\
\hline $\mathrm{T}_{17}\left(\mathrm{~T}_{10}+\mathrm{T}_{7}\right)$ & $12.11 \mathrm{~d}-\mathrm{g}$ & 9.98 b-e & $2.14 \mathrm{e}-\mathrm{h}$ & $0.654 \mathrm{c}-\mathrm{f}$ & $0.036 \mathrm{~d}-\mathrm{f}$ & $0.054 \mathrm{~cd}$ & 0.004 & $0.697 \mathrm{c}-\mathrm{f}$ \\
\hline $\mathrm{T}_{18}\left(\mathrm{~T}_{10}+\mathrm{T}_{8}\right)$ & $12.25 \mathrm{~d}-\mathrm{f}$ & 9.89 b-f & $2.36 \mathrm{~d}-\mathrm{f}$ & $0.745 \mathrm{c}-\mathrm{e}$ & $0.043 \mathrm{c}-\mathrm{e}$ & $0.062 \mathrm{bc}$ & 0.004 & $0.531 \mathrm{e}-\mathrm{g}$ \\
\hline $\mathrm{T}_{19}\left(\mathrm{~T}_{10}+\mathrm{T}_{9}\right)$ & $12.14 \mathrm{~d}-\mathrm{g}$ & $9.73 \mathrm{~b}-\mathrm{f}$ & $2.41 \mathrm{de}$ & $0.721 \mathrm{c}-\mathrm{f}$ & 0.039 c-e & $0.057 \mathrm{bc}$ & 0.003 & $0.760 \mathrm{~b}-\mathrm{e}$ \\
\hline $\mathrm{T}_{20}$ (Untreated Control) & $10.40 \mathrm{j}$ & 9.55 b-f & $0.85 \mathrm{j}$ & $0.321 \mathrm{~h}$ & $0.012 \mathrm{~g}$ & $0.023 \mathrm{f}$ & 0.001 & $0.333 \mathrm{~g}$ \\
\hline $\operatorname{LSD}(P \leq 0.05)$ & 0.6003 & 0.579 & 0.278 & 0.163 & 0.016 & 0.016 & NS & 0.225 \\
\hline
\end{tabular}

Values within the same column having a common letter(s) do not differ significantly $(P \leq 0.05) \quad$ NS= Non Significant 
Table 4. Effect of different treatments on some growth characters of chilli seedlings

\begin{tabular}{|c|c|c|c|c|c|c|c|c|}
\hline Treatments & $\begin{array}{l}\text { Seedling } \\
\text { height }\end{array}$ & $\begin{array}{l}\text { Shoot } \\
\text { length }\end{array}$ & Root length & $\begin{array}{l}\text { Fresh shoot } \\
\text { weight }\end{array}$ & $\begin{array}{c}\text { Fresh root } \\
\text { weight }\end{array}$ & $\begin{array}{l}\text { Dry shoot } \\
\text { weight }\end{array}$ & $\begin{array}{c}\text { Dry root } \\
\text { weight }\end{array}$ & Biomass \\
\hline $\mathrm{T}_{1}$ (Soil application of $T$. harzianum) & $18.37 \mathrm{~d}$ & $13.63 \mathrm{~cd}$ & $4.73 \mathrm{f}$ & $2.10 \mathrm{e}$ & $0.185 \mathrm{f}$ & $0.397 \mathrm{e}$ & $0.020 \mathrm{~d}$ & $2.28 \mathrm{~d}$ \\
\hline $\mathrm{T}_{2}$ (Soil application of Poultry Refuse) & $19.30 \mathrm{c}$ & $14.03 \mathrm{bc}$ & $5.27 \mathrm{~cd}$ & $2.57 \mathrm{~b}$ & $0.227 \mathrm{c}$ & $0.420 \mathrm{~d}$ & $0.040 \mathrm{~b}$ & $2.77 \mathrm{~b}$ \\
\hline $\mathrm{T}_{3}$ (Soil application of Cocodust) & $16.80 \mathrm{~h}$ & $12.37 \mathrm{fg}$ & $4.43 \mathrm{~h}$ & $1.49 \mathrm{~h}$ & $0.155 \mathrm{hi}$ & $0.244 \mathrm{k}$ & $0.010 \mathrm{e}$ & $1.64 \mathrm{gh}$ \\
\hline $\mathrm{T}_{4}$ (Soil application of Vermicompost) & $17.63 \mathrm{e}$ & $12.93 \mathrm{e}$ & $4.70 \mathrm{fg}$ & $2.350 \mathrm{~cd}$ & $0.215 \mathrm{~d}$ & $0.352 \mathrm{~g}$ & $0.03 \mathrm{c}$ & $2.563 \mathrm{c}$ \\
\hline $\mathrm{T}_{5}$ (Soil application of Ash) & $16.40 \mathrm{i}$ & $12.13 \mathrm{gh}$ & $4.27 \mathrm{hi}$ & $1.20 \mathrm{i}$ & $0.145 \mathrm{j}$ & $0.208 \mathrm{~m}$ & $0.010 \mathrm{e}$ & $1.34 \mathrm{j}$ \\
\hline $\mathrm{T}_{6}$ (Soil application of Sawdust) & $15.80 \mathrm{j}$ & $11.70 \mathrm{~h}-\mathrm{j}$ & $4.10 \mathrm{ij}$ & $1.08 \mathrm{i}$ & $0.128 \mathrm{k}$ & $0.277 \mathrm{~h}$ & $0.004 \mathrm{e}-\mathrm{g}$ & $1.21 \mathrm{k}$ \\
\hline $\mathrm{T}_{7}$ (Soil application of Khudepana) & $16.13 \mathrm{i}$ & $11.80 \mathrm{hi}$ & $4.33 \mathrm{hi}$ & $1.0 \mathrm{i}$ & $0.112 \mathrm{~m}$ & $0.129 \mathrm{p}$ & $0.006 \mathrm{e}-\mathrm{g}$ & $1.18 \mathrm{k}$ \\
\hline $\mathrm{T}_{8}$ (Soil application of Cowdung) & $16.90 \mathrm{gh}$ & $12.53 \mathrm{e}-\mathrm{g}$ & $4.37 \mathrm{~h}$ & $1.45 \mathrm{~h}$ & $0.128 \mathrm{k}$ & 0.163 o & $0.006 \mathrm{e}-\mathrm{g}$ & $1.58 \mathrm{hi}$ \\
\hline $\mathrm{T}_{9}$ (Soil application of Solarized Sand) & $15.70 \mathrm{j}$ & $11.47 \mathrm{ij}$ & $4.233 \mathrm{hi}$ & $1.39 \mathrm{~h}$ & $0.154 \mathrm{i}$ & 0.2231 & $0.008 \mathrm{ef}$ & $1.54 \mathrm{hi}$ \\
\hline $\mathrm{T}_{10}($ Seed treatment of $T$. harzianum $)$ & $15.20 \mathrm{k}$ & $11.27 \mathrm{j}$ & $3.93 \mathrm{j}$ & $1.06 \mathrm{i}$ & 0.1171 & $0.118 \mathrm{q}$ & $0.003 \mathrm{fg}$ & $1.18 \mathrm{k}$ \\
\hline $\mathrm{T}_{11}\left(\mathrm{~T}_{10}+\mathrm{T}_{1}\right)$ & $20.30 \mathrm{~b}$ & $14.37 \mathrm{~b}$ & $5.93 \mathrm{~b}$ & $2.32 \mathrm{~d}$ & $0.201 \mathrm{e}$ & $0.458 \mathrm{c}$ & $0.030 \mathrm{c}$ & $2.52 \mathrm{c}$ \\
\hline $\mathrm{T}_{12}\left(\mathrm{~T}_{10}+\mathrm{T}_{2}\right)$ & $21.40 \mathrm{a}$ & $15.17 \mathrm{a}$ & $6.23 \mathrm{a}$ & $2.83 \mathrm{a}$ & $0.295 \mathrm{a}$ & $0.817 \mathrm{a}$ & $0.050 \mathrm{a}$ & $3.12 \mathrm{a}$ \\
\hline $\mathrm{T}_{13}\left(\mathrm{~T}_{10}+\mathrm{T}_{3}\right)$ & $18.43 \mathrm{~d}$ & $13.37 \mathrm{~d}$ & $5.07 \mathrm{de}$ & $1.96 \mathrm{fg}$ & $0.182 \mathrm{f}$ & $0.267 \mathrm{i}$ & $0.020 \mathrm{~d}$ & $2.14 \mathrm{ef}$ \\
\hline $\mathrm{T}_{14}\left(\mathrm{~T}_{10}+\mathrm{T}_{4}\right)$ & $19.07 \mathrm{c}$ & $13.63 \mathrm{~cd}$ & $5.433 \mathrm{c}$ & $2.47 \mathrm{bc}$ & $0.244 \mathrm{~b}$ & $0.531 \mathrm{~b}$ & $0.040 \mathrm{~b}$ & $2.71 \mathrm{~b}$ \\
\hline $\mathrm{T}_{15}\left(\mathrm{~T}_{10}+\mathrm{T}_{5}\right)$ & $17.23 \mathrm{fg}$ & $12.33 \mathrm{fg}$ & 4.90 ef & $1.46 \mathrm{~h}$ & $0.160 \mathrm{~h}$ & $0.255 \mathrm{j}$ & $0.020 \mathrm{~d}$ & $1.72 \mathrm{~g}$ \\
\hline $\mathrm{T}_{16}\left(\mathrm{~T}_{10}+\mathrm{T}_{6}\right)$ & $17.20 \mathrm{fg}$ & $12.73 \mathrm{ef}$ & $4.47 \mathrm{gh}$ & $1.19 \mathrm{i}$ & $0.147 \mathrm{j}$ & $0.180 \mathrm{n}$ & $0.006 \mathrm{e}-\mathrm{g}$ & $1.33 \mathrm{j}$ \\
\hline $\mathrm{T}_{17}\left(\mathrm{~T}_{10}+\mathrm{T}_{7}\right)$ & 17.47 ef & $12.53 \mathrm{e}-\mathrm{g}$ & $4.93 \mathrm{ef}$ & $1.35 \mathrm{~h}$ & $0.126 \mathrm{k}$ & $0.131 \mathrm{p}$ & $0.010 \mathrm{e}$ & $1.47 \mathrm{i}$ \\
\hline $\mathrm{T}_{18}\left(\mathrm{~T}_{10}+\mathrm{T}_{8}\right)$ & $17.53 \mathrm{ef}$ & $12.53 \mathrm{e}-\mathrm{g}$ & $5.00 \mathrm{e}$ & $1.90 \mathrm{~g}$ & $0.143 \mathrm{j}$ & $0.180 \mathrm{n}$ & $0.009 \mathrm{e}$ & $2.04 \mathrm{f}$ \\
\hline $\mathrm{T}_{19}\left(\mathrm{~T}_{10}+\mathrm{T}_{9}\right)$ & $16.73 \mathrm{~h}$ & $11.87 \mathrm{hi}$ & $4.87 \mathrm{ef}$ & $2.06 \mathrm{ef}$ & $0.170 \mathrm{~g}$ & $0.357 \mathrm{f}$ & $0.010 \mathrm{e}$ & $2.23 \mathrm{de}$ \\
\hline $\mathrm{T}_{20}$ (Untreated Control) & 14.171 & $10.80 \mathrm{k}$ & $3.37 \mathrm{k}$ & $0.83 \mathrm{j}$ & $0.107 \mathrm{~m}$ & $0.103 \mathrm{r}$ & $0.001 \mathrm{~g}$ & 0.9401 \\
\hline $\operatorname{LSD}(P \leq 0.05)$ & 0.3185 & 0.4260 & 0.2368 & 0.1266 & 0.005167 & 0.005167 & 0.00517 & 0.1266 \\
\hline
\end{tabular}

Values within the same column having a common letter(s) do not differ significantly $(P \leq 0.05)$ 
The highest fresh shoot weight and biomass was recorded in $\mathrm{T}_{11}$ which was closely followed by $\mathrm{T}_{12}$. The lowest growth characters were recorded in control treatment. Vermicompost also showed better performance than other soil amendment in terms of all growth characters of potato seedlings. Sawdust, khudepana, cocodust and cowdung showed significantly similar effect on growth characters of potato seedlings. From this experiment, it was found that all the growth characters were recorded higher when seeds were sown with different soil amendments after treated with $T$. harzianum than without treated with $T$. harzianum. Almost similar results were also found in case of chilli seedlings (Table 4). The highest seedling height, shoot length and root length were recorded in $T_{12}$ followed by $T_{11}$, $\mathrm{T}_{2}, \mathrm{~T}_{14}$ and $\mathrm{T}_{13}$ while the lowest data were found in control plot. $\mathrm{T}_{12}$ (Seed treated with $T$. harzianum + soil application of poultry refuse) showed the best growth characters of chilli seedlings regarding fresh shoot weight, fresh root weight, dry shoot weight, dry root weight and biomass.

The findings of the present study are the satisfactory agreement with the findings of other researchers (Roy et al., 1998; Manoranjitham et al., 2000; Jayaraj et al., 2006; Faruq and Islam, 2007). Roy et al. (1998) found that seed treatments of Trichoderma viride, T. harzianum and T. koningii reduced damping off of cabbage caused by Rhizoctonia solani in both sterilised and unsterilised soil. Treating cabbage seeds with a spore suspension of $T$. viride and $T$. harzianum separately was more effective than the application of antagonist to the soil at the inoculum rate of $2.0 \%(\mathrm{w} / \mathrm{w})$. The combination of seed treatment and soil application with Trichoderma spp. further reduced the pre- and post-emergence damping off of cabbage in the nursery. Manoranjitham et al. (2000) reported that Soil application of $T$. viride and $P$. fluorescens effectively controlled the preemergence and post-emergence damping off of tomato and increased the shoot length, root length and dry matter production of tomato seedlings. Jayaraj et al. (2006) reported that the seed treatment with $T$. harzianum formulations reduced the incidence of damping-off disease of tomato by up to $74 \%$ and enhanced plant biomass under greenhouse and field (Tamil Nadu, India) conditions. Faruq and Islam (2007) reported that poultry refuse reduced damping off incidence by $90.56 \%$ (30 DAS) in eggplant and $84.81 \%$ (25 DAS) in tomato seedlings over control and increased seedlings growth characters like shoot length, root length, seedling height, fresh shoot weight, fresh root weight and fresh seedling weight of eggplant and tomato. They also reported that application of neem compost and vermicompost also showed promising effect against damping off disease and also enhanced seed germination and growth characters of eggplant and tomato seedlings.

\section{Conclusions}

The findings of the present studies concluded that seed treatment with Trichoderma harzianum along with soil amendment of poultry refuse and vermicompost offered better performance against damping off disease of potato and chilli. Seed treatment with $T$. harzianum followed by soil amendment might be suggested to control damping off disease complex of potato and chilli.

\section{References}

Anonymous. 1960. BARI (Bangladesh Agricultural Research Institute) Annual report (1960-1961), BARI, Joydebpur, Gazipur. 75 p.

Anonymous. 1966. Index of Plant virus diseases. Agricultural Hand book. No. 307. U.S. Department of Agriculture. USA. 446 p.

BARC. 1997. Fertilizer Recommendation Guide. Bangladesh Agric. Res. Council, Dhaka. 1-29 pp.

BBS. 2009. Year Book of Agricultural Statistics of Bangladesh, 2008-09. Statistics Division, All Crops Summary (2008-2009) Dhaka, 6-7 pp.

Begum, M. M., Rahman, M. A. and Hossain, I. 1999. Antagonistic effect Trichoderma 
harzianum on Sclerotium rolfsii in food legumes. Bangladesh Journal of Bio Science, 7:81-88.

Chaudhury, E. H., Sarker, M. H. and Rashid, M. H. 1987. Agronomic management for potato production from true seed in Bangladesh. Proceedings of the workshop on true potato seed research in Bangladesh. PRC, BARI, Joydebpur, Gazipur, 18-27 pp.

Chowdhury, M. S. M., Hossain, I., Fakir, G. A., Aminuzzaman, F. M. and Islam, M. R. 2000. Tolerance of Trichoderma harzianum and Gliocladium viride agrochemical and their antagonistic effect on seed borne mycoflora of pigeon pea. Bangladesh Journal of Seed Science \& Technology, 4(1\&2):83-86.

Das, A. C. 1984. Effect of cultural practices on the Damping off incidence of TPS seedlings and its chemical control invitro. M. Sc. Ag. Thesis, Bangladesh Agricultural University. $50 \mathrm{pp}$.

Dey, T. K. 2005. Effect of soil solarization in controlling damping off disease of true potato seedlings (TPS). Bangladesh Journal of Plant Pathology, 21(1\&2): 93.

Elango, F. 1986. The role of high soil temperature in the damping off of true potato seedlings in the low land tropics. Tropical Agriculture (Trinidad), 63: 66-67.

Faruq, A. N. and Islam, M. T., 2007. Effect of selected soil amendments on seed germination, seedling growth and control of damping off of eggplant and tomato seedlings. Journal of Agricultural Education and Technology, 10(1\&2): 4348.

Gammarra, D., Torres, H. and Martin, C. 1986. Pythium splendens y $P$. deliense causantes de damping off plantulus de papa sen san Ramon, Peru, Fitopatologia, 21:5.

Grinstein, A., Katan, J., Razik, A., Zeydan, A. and Elad, Y. 1986. Control of Sclerotium rolfsii and weeds in peanuts by solar heating of soil. Plant Disease Reports, 63:1056-1059.

Hilocks, R. J. and Waller, J. M. 1997. Soil borne diseases of tropical crops, (ed.). CAB International, New York, USA. 377-397 pp.

Hossain, I. and Naznin, M. H. A. 2005. BAUBiofengicide in controlling seedling diseases of summer vegetables. $B A U$ Research Progress, 15:35.

Hossain, I. and Shamsuzzaman. 2003. Developing Trichoderma based biofungicide using agro-wastes. $B A U$ Research Progress, 14: 149-50.

Islam, M. T. and Faruq, A. N. 2008. Effect of selected soil amendments on seed germination, seedling growth and control of damping off of chilli seedlings. Journal of Sher-e-Bangla Agricultural University, 2(2): 12-16.

Islam, M. T., Islam, M. R., Aminuzzaman, F. M., and Yesmin, S. 2007. Management of Damping off of Vegetable seedlings through some selected Soil amendments and Chemicals. Journal of Agricultural Science and Technology, 8(2): 27-31.

Jayaraj, J., Radhakrishnan, N. V., Velazhahan, R. 2006. Development of formulations of Trichoderma harzianum strain M1 for control of damping-off of tomato caused by Pythium aphanidermatum. Phytopathology and Plant Protection, 39(1): 1-8.

Jones, R. A. C. 1982. Test for transmission of four viruses through potato true seed. Annual of Applied Biology, 100: 315-320.

Manoranjitham, S. K., Prakasan, V., Rajappan, K., and Amutha, G. 2000. Effect of two antagonists on damping off disease of tomato. Indian Phytopathology, 53 (4): 441-443.

Mukhopadhyay, A. N., 1995. Exploitation of Trichoderma harzianum and Gliocladium virens for biological seed treatment against seed borne disease. Indian Journal of 
Mycology and Plant Pathology, 2(1\&2):124.

Martin, C. and Torres, H. 1989. Control of Rhizoctonia and other soil borne disease of TPS. In Fungal disease of Potato. CIP, Lima, Peru. 191-205 pp.

Prasad, C. S. and Gupta, V. 2002. Studies on bio-efficacy of Trichoderma harzianum in management of stem rot of potato (Rhizoctonia solani). National Academy Science Letters, 25(11/12): 357-359.

Rangasami, G. 1988. Diseases of crop plants in India. Printice-Hall of India Private Ltd. New Delhi. 101p.

Rashid, M. M. 1987. Problems of production of true potato seed in Bangladesh. Proceedings of the workshop on true potato seed research in Bangladesh. 5-7 pp.
Roy, S. J., Das, B. S. and Bora, L. C. 1998. Non pesticidal management of damping off of cabbage caused by Rhizoctonia solani kuehn. Journal of the Agricultural Science Society of North East India, 11(2): 127-130.

Szczech, M. M. 1999. Suppressiveness of vermicompost against Fusarium wilt of tomato. Journal of Phytopathology, 147(3): 155-161.

Torres, H. 1989. Soil borne and foliar diseases in the high land tropics. Fungal diseases of potato. International Potato Centre. 169179 pp.

Yeasmin. R. 2004. Integrated management of seedling diseases of blackgram, mungbean and lentil. M. S. thesis. Department Plant Pathology, Bangladesh Agricultural University, Mymenshing, Bangladesh. 7273 pp. 
